\title{
Cytoskelett
}

\section{Einblicke in die Entstehung von Mikrotubuli}

STEFAN PFEFFER, ELMAR SCHIEBEL

ZENTRUM FÜR MOLEKULARE BIOLOGIE DER UNIVERSITÄT HEIDELBERG (ZMBH), HEIDELBERG

Microtubules are part of the cytoskeleton and promote various essential cellular functions. Microtubules are dynamic polymers composed of heterodimeric $\alpha / \beta$-tubulin subunits and can assemble de novo in a 'structural templating' mechanism assisted by ring-like complexes containing the protein $\gamma$-tubulin. Recent cryo-electron microscopy structures of such $\gamma$-tubulin ring complexes from vertebrates propelled our understanding of their architecture, assembly and activation mechanism.

DOI: $10.1007 / \mathrm{s} 12268-020-1341-2$

(C) Die Autoren 2020

Mikrotubuli sind Teil des Cytoskeletts und haben essenzielle Funktionen bei der mitotischen und meiotischen Chromosomenteilung, der Zellorganisation und bei vielen intrazellulären Transportvorgängen. Mikrotubuli sind zylindrische Proteinkomplexe mit einem Durchmesser von 25 Nanometern und einer Länge von bis zu 50 Mikrometern. Sie bestehen aus vielen Kopien des Heterodimers $\alpha / \beta$-Tubulin, die in 13 geordneten Reihen, den Protofilamenten, angeordnet sind und so die Wandung des Zylinders bilden (Abb. 1). Die $\alpha / \beta$-Tubulin-Dimere innerhalb eines Mikrotubulus haben alle die gleiche Orientierung und verleihen den Mikrotubuli dadurch eine eindeutige Polarität. Mikrotubuli sind sehr dynamische Strukturen, und vor allem das Plusende der Mikrotubuli kann durch Hinzufügen oder Entfernen von $\alpha / \beta$ Tubulin-Untereinheiten verlängert oder verkürzt werden [1]. Besonders die Anzahl und Länge der Mikrotubuli sowie deren Ausrichtung innerhalb der Zelle können genau reguliert werden.

\section{Bäckerhefe besitzt ein minimales System für die Mikrotubuli- Nukleation}

Schon vor fast 30 Jahren wurde das Protein $\gamma$-Tubulin als essenzieller Faktor für die

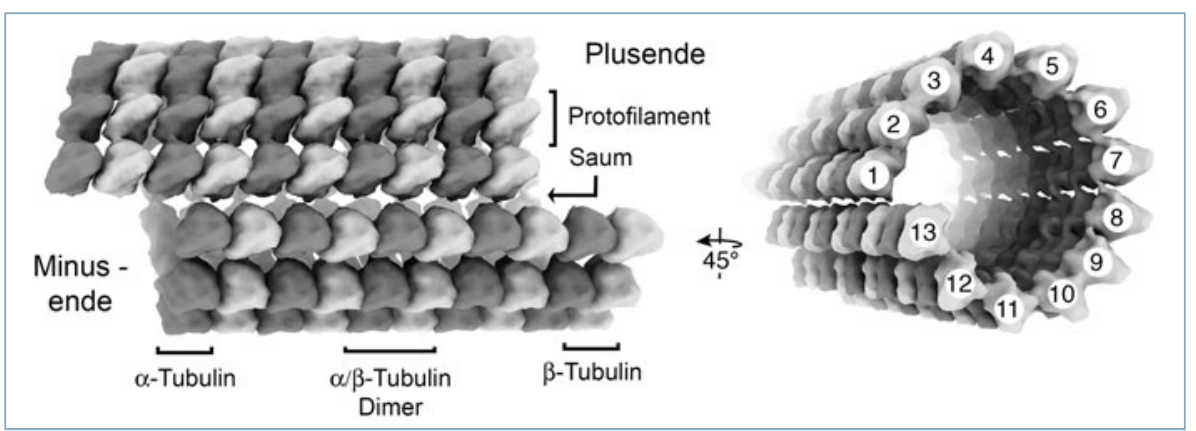

$\Delta$ Abb. 1: Schematische Darstellung des Aufbaus von Mikrotubuli (MT). $\alpha / \beta$-Tubulin-Untereinheiten sind in 13 Reihen, den Protofilamenten, angeordnet und bilden die Wandung des MT-Zylinders. Die ersten und letzten Protofilamente sind durch einen Saum getrennt. $\alpha$-Tubulin (dunkelgrau) bildet das Minusende, während $\beta$-Tubulin (hellgrau) das dynamische Plusende abschließt.
Assemblierung von Mikrotubuli aus $\alpha / \beta$ Tubulin-Untereinheiten identifiziert [2]. Es wurde jedoch schnell klar, dass $\gamma$-Tubulin diese Funktion nicht allein, sondern im Zusammenwirken mit anderen Proteinen im Kontext eines größeren Proteinkomplexes erfüllt [3] Mithilfe von genetischen Suppressorstudien in der Bäckerhefe Saccharomyces cerevisiae wurden dann die Gene GCP2 und GCP3 als $\gamma$-Tubulin-Interaktoren identifiziert. GCP2 und GCP3 codieren für paraloge Proteine, die zusammen mit zwei $\gamma$-Tubulin-Untereinheiten den kleinen $\gamma$-Tubulin-Komplex $(\gamma$-tubulin small complex, $\gamma$-TuSC) bilden [4]. Strukturelle Untersuchungen unter Verwendung von Negativkontrast-Elektronenmikroskopie zeigten eine Y-ähnliche Anordnung der Untereinheiten, bei der die C-Termini von GCP2 und GCP3 jeweils mit einem $\gamma$-Tubulin-Molekül interagieren, während die N-Termini von GCP2 und GCP3 aneinanderbinden und so den $\gamma$-TuSC zusammenhalten (Abb. 2A, [5]).

Untersuchungen zeigten, dass der $\gamma$-TuSC mit dem N-Terminus des Proteins Spc110 wechselwirken muss, um in einen mikrotubulinukleationskompetenten Zustand überführt zu werden [6]. Wie von K. Sawin beschrieben, besitzen Spc110 und weitere ähnliche Proteine ein kurzes gemeinsames Motiv, das als centrosomin motif 1 (CM1) bezeichnet wurde [7]. Die Kryo-Elektronenmikroskopie (Kryo-EM) zeigte, dass die Bindung von CM1 eine Oligomerisierung des $\gamma$-TuSC induziert, wobei eine linkshändige $\gamma$-TuSC-Helix entsteht (Abb. 2B, [8]). Die dreidimensionale Anordnung von $\gamma$-TubulinUntereinheiten in dieser Helix ist vergleichbar mit der Anordnung von $\alpha / \beta$-TubulinUntereinheiten in einem Mikrotubulus. Basierend auf dieser Beobachtung und der bereits bekannten Interaktion zwischen $\alpha$-Tubulin und $\gamma$-Tubulin wurde ein inzwischen weithin akzeptiertes Modell für die Mikrotubuli-Nukleation in Hefe vorgeschlagen, bei dem eine Helix aus sieben Kopien des $\gamma$-TuSC und Spc110 als optimale strukturelle Vorlage für die Assemblierung eines neuen Mikrotubulus aus $\alpha / \beta$-TubulinUntereinheiten fungiert (Abb. 2C). 


\section{Das Mikrotubuli-Nukleationssystem gewinnt in anderen Organismen an Komplexität}

Das minimale Mikrotubuli-Nukleationssystem der Bäckerhefe, bestehend aus $\gamma$-TuSC und CM1-Proteinen, ist relativ einfach aufgebaut. Bereits in der verwandten Hefe Candida albicans wird ein zusätzliches essenzielles Protein, Mozart (Mzt1), für die Assemblierung einer $\gamma$-TuSC-Helix benötigt. In der Spalthefe Schizosaccharomyces pombe kommen weitere Komponenten hinzu. Neben den $\gamma$-TuSC-Komponenten und CM1-Proteinen wurden weitere zu GCP2 und GCP3 verwandte Proteine entdeckt, die als GCP4, GCP5 und GCP6 bezeichnet werden. Eine ähnliche Ausstattung an GCP-Genen gibt es auch in Aspergillus nidulans, Drosophila melanogaster, dem Krallenfrosch Xenopus laevis und in menschlichen Zellen. In S. pombe, A. nidulans und D. melanogaster ist die Mikrotubuli-Assemblierung über CM1-Proteine und $\gamma$-TuSCOligomere essenziell. Die GCP4-6-Gene scheinen in diesen Organismen dagegen eine eher untergeordnete Bedeutung zu haben. Im Gegensatz dazu reicht das minimale $\gamma$-TuSC/ CM1-System in Vertebraten überraschenderweise nicht für die Mikrotubuli-Nukleation aus. Diese übernimmt ausschließlich der im Aufbau wesentlich komplexere $\gamma$-TubulinRingkomplex ( $\gamma$-TuRC), der aus den Proteinen GCP2-6, $\gamma$-Tubulin, der regulierenden Kinase NME7 und den Proteinen Mozart 1 und 2 besteht.

\section{Kryo-EM-Studien klären die Struktur des komplexen $\gamma$-TuRC aus Vertebraten auf}

Es war zwar bekannt, dass die verschiedenen GCP-Varianten im $\gamma$-TuRC in unterschiedlicher Kopienzahl vorkommen, jedoch war unklar, ob die relative Stöchiometrie und Anordnung der GCP-Varianten in allen $\gamma$-TuRC-Komplexen identisch ist. Weiterhin gab es keine Informationen darüber, welche Funktionen die einzelnen GCP-Moleküle im $\gamma$-TuRC erfüllen und ob das Template-Modell der $\gamma$-TuSC-Helix aus Bäckerhefe direkt auf den $\gamma$-TuRC übertragen werden kann. Um die Beantwortung dieser offenen Fragen voranzutreiben, wurden bereits seit dem Jahr 2000 Versuche unternommen, die Struktur des $\gamma$-TuRC aus höheren Eukaryoten durch KryoEM zu beschreiben [9]. Es kam jedoch erst im Jahr 2019 zum entscheidenden Durchbruch als die Struktur des humanen und $X$. laevis- $\gamma$-TuRC beschrieben wurde [10-12]. Diese Studien zeigten, dass der $\gamma$-TuRC die
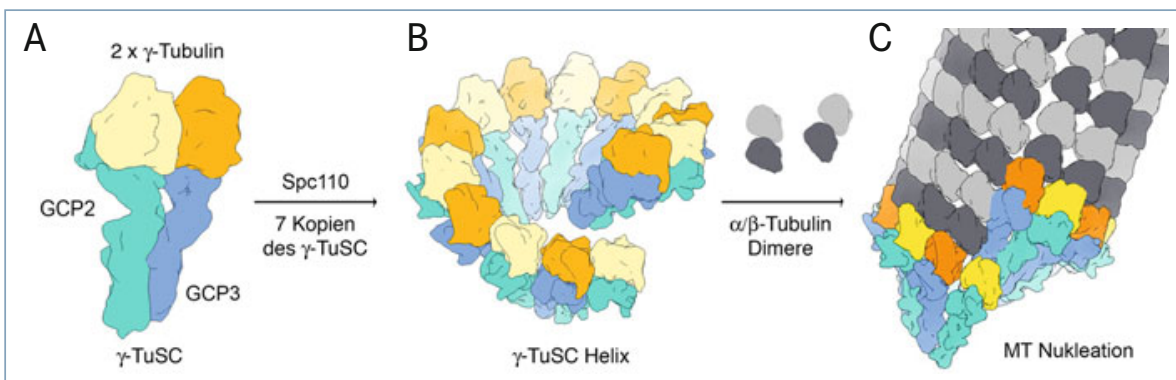

$\Delta$ Abb. 2: Schematische Darstellung der Mikrotubuli-Nukleation durch den $\gamma$-tubulin small complex ( $\gamma$-TuSC) in Hefe. A, Zwei Moleküle $\gamma$-Tubulin und jeweils ein Molekül GCP2 und GCP3 formen den $\gamma$-TuSC. B, Die Interaktion des $\gamma$-TuSC mit dem CM1-Motiv in Spc 110 führt zur Oligomerisierung von mehreren Kopien des $\gamma$-TuSC. In der entstehenden linkshändigen Helix wechseln sich GCP2 und GCP3 ab. C, Diese Anordnung dient als strukturelle Vorlage für die Assemblierung eines Mikrotubulus (MT) aus $\alpha / \beta$-Tubulin-Untereinheiten.

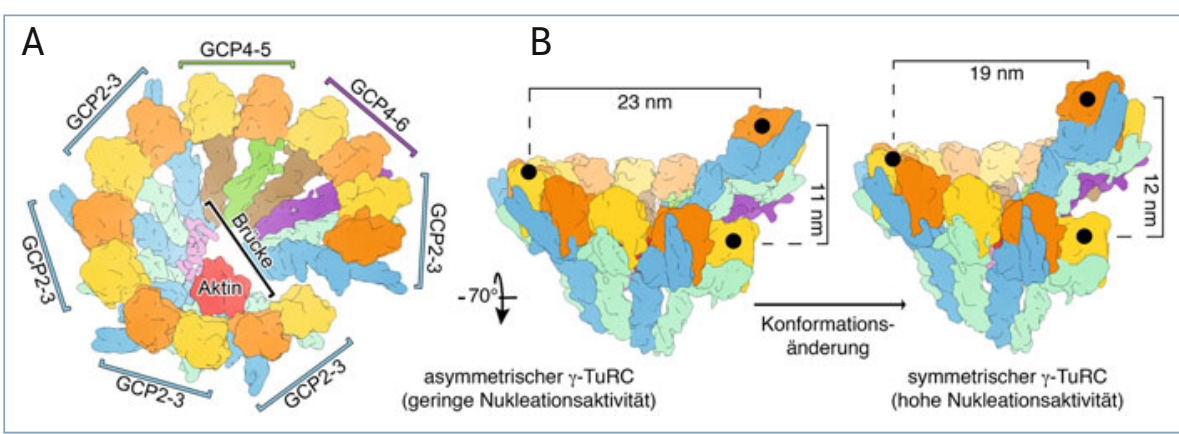

$\triangle$ Abb. 3: Schematische Darstellung der Struktur und Aktivierung des $\gamma$-TuRC in Vertebraten. A, Jeweils 14 GCP- und $\gamma$-Tubulin-Untereinheiten bilden die $\gamma$-TuRC-Spirale. Der Komplex wird durch eine strukturelle Brücke auf der Innenseite des $\gamma$-TuRC stabilisiert, die eine Kopie des Proteins Aktin enthält. B, Um als strukturelle Vorlage für die Assemblierung eines Mikrotubulus dienen zu können, muss die $\gamma$-TuRC-Spirale durch eine Konformationsänderung verengt werden.

Form einer offenen, linkshändigen Spirale aus jeweils 14 GCP- und $\gamma$-Tubulin-Untereinheiten annimmt. In allen $\gamma$-TuRC-Komplexen liegen die GCP-Untereinheiten in gleicher Abfolge und mit definierter Stöchiometrie vor. Die GCP-Moleküle sind in Paaren mit $\gamma$-TuSC-ähnlicher Konfiguration angeordnet: Auf vier GCP(2-3)-Dimere folgen ein GCP(4-5)- und ein GCP(4-6)-Dimer, und ein letztes GCP(2-3)-Dimer schließt den $\gamma$-TuRC ab (Abb. 3A). Überraschenderweise zeigten alle Studien, dass sich eine strukturelle Brücke an der Innenseite der $\gamma$-TuRC-Spirale entlangzieht, deren fester Bestandteil auch ein Aktin-ähnliches Protein ist. Experimente zeigten, dass es sich dabei tatsächlich um kanonisches Aktin handelt, das tief in die Struktur des $\gamma$-TuRC integriert ist [10]. Weiterführende Experimente sprechen gegen eine Funktion dieses Aktinmoleküls in der Nukleation von Aktinfilamenten, deuten aber auf eine funktionelle Rolle bei der Mikrotubuli-Nukleation hin.

Es stellt sich die Frage, wie eine derart komplexe Struktur immer in identischer
Weise in der Zelle assembliert werden kann. Experimentelle Daten zeigen, dass eine lange Insertionsdomäne in GCP6 wahrscheinlich als Bauplan für den $\gamma$-TuRC fungiert, indem sie spezifisch an die N-Termini von ausgewählten GCP-Proteinen bindet und so deren Reihenfolge im $\gamma$-TuRC definiert. Im Gegensatz zur $\gamma$-TuSC-Helix der Bäckerhefe ist der vollständig assemblierte $\gamma$-TuRC keine optimale strukturelle Vorlage für die Mikrotubuli-Assemblierung. Nur durch eine Verengung der $\gamma$-TuRC-Spirale über eine konzertierte Konformationsänderung kann ein „perfektes“ Template erreicht werden (Abb. 3B).

\section{Literatur}

[1] Borisy G, Heald R, Howard J et al. (2016) Microtubules: 50 years on from the discovery of tubulin. Nat Rev Mol Cell Biol 17:322-328

[2] Oakley BR, Oakley E, Yoon Y et al. (1990) $\gamma$-Tubulin is a component of the spindle pole body that is essential for microtubule function in Aspergillus nidulans. Cell 61:1289-1301 [3] Zheng Y, Wong ML, Alberts B et al. (1995) Nucleation of microtubule assembly by a gamma-tubulin-containing ring complex. Nature 378:578-583

[4] Knop M, Schiebel E (1997) Spc98p and Spc97p of the yeast gamma-tubulin complex mediate binding to the spindle pole body via their interaction with Spc110p. EMBO J 16:6985-6995 
[5] Kollman JM, Zelter A, Muller EG et al. (2008) The structure of the $\gamma$-tubulin small complex: implications of its architecture and flexibility for microtubule nucleation. Mol Biol Cell 19:207-215

[6] Knop M, Schiebel E (1998) Receptors determine the cellular localization of a $\gamma$-tubulin complex and thereby the site of microtubule formation. EMBO J 17:3952-3967

[7] Samejima I, Miller VJ, Groocock LM el al. (2008) Two distinct regions of Mto1 are required for normal microtubule nucleation and efficient association with the $\gamma$-tubulin complex in vivo. J Cell Sci 121:3971-3980

[8] Kollman JM, Polka JK, Zelter A et al. (2010) Microtubule nucleating gamma-TuSC assembles structures with 13-fold microtubule-like symmetry. Nature 466:879-882

[9] Moritz M, Braunfeld MB, Guenebaut V et al. (2000)

Structure of the gamma-tubulin ring complex: a template for microtubule nucleation. Nat Cell Biol 2:365-370

[10] Liu P, Zupa E, Neuner A et al. (2019) Insights into the assembly and activation of the microtubule nucleator gammaTuRC. Nature, doi: 10.1038/s41586-019-1896-6

[11] Wieczorek M, Urnavicius L, Ti SC et al. (2020) Asymmetric molecular architecture of the human gammatubulin ring complex. Cell 180:165-175

[12] Consolati T, Locke J, Roostalu J et al. (2019) Microtubule nucleation by single human $\gamma \mathrm{TuRC}$ in a partly open asymmetric conformation. BioRxiv, https://doi.org/10.1101/853218
Funding: Open Access funding provided by Projekt DEAL

Open Access: Dieser Artikel wird unter der Creative Commons Namensnennung 4.0 International Lizenz veröffentlicht, welche die Nutzung, Vervielfältigung, erlabt, sofern Sie den/die ursprünglichen Autor(en) und die Ouelle ordnungscmä $B$ nennen, inen Link zur Creative Commons Lizenz beifügen und an enthaltenen Bilder und sonstiges Drittmaterial unterliegen ebenfalls genannten Creative Commons Lizenz, sofern sich aus der Abbildungslegende nichts anderes ergibt. Sofern das betreffende Material nicht unter der genannten Creative Commons Lizenz steht und die betreffende Handlung nich nach gesetzlichen Vorschriften erlaubt ist, ist für die oben aufgeführten Weiterverwendungen des Materials die Einwiligung des jeweiligen Rechteinhabers einzuholen. Weitere Details zur Lizenz entnehmen Sie bitte der
Korrespondenzadresse:

Dr. Stefan Pfeffer

Prof. Dr. Schiebel

Zentrum für molekulare Biologie $(\mathrm{ZMBH})$

der Universität Heidelberg

Im Neuenheimer Feld 282

D-69120 Heidelberg

s.pfeffer@zmbh.uni-heidelberg.de

e.schiebel@zmbh.uni-heidelberg.de

$\begin{aligned} & \text { Stefan Pfeffer } \\ & \text { Jahrgang 1984. Biochemiestudium an der Universität Tübingen. } 2015 \text { Promotion an } \\ & \text { der TU München. 2015-2018 Postdoktorand am Max-Planck-Institut für Biochemie, } \\ & \text { Martinsried. Seit 2018 unabhängiger Nachwuchsgruppenleiter am Zentrum für } \\ & \text { Molekulare Biologie der Universität Heidelberg (ZMBH). }\end{aligned}$
$\begin{aligned} & \text { Elmar Schiebel } \\ & \text { Jahrgang 1960. Biochemiestudium an der Universität Tübingen und am University } \\ & \text { College London, UK. 1989 Promotion an der Universität Tübingen. 1989-1991 Post- } \\ & \text { doktorand an der University of California, USA. 1991-1997 Gruppenleiter am Max- } \\ & \text { Planck-Institut für Biochemie, Martinsried, 1997-2001 am Beatson Institute for Can- } \\ & \text { cer Research, UK, und 2001-2005 am Paterson Institute for Cancer Research, UK. } \\ & \text { Seit 2005 Professor am Zentrum für Molekulare Biologie der Universität Heidelberg } \\ & \text { (ZMBH). }\end{aligned}$

\section{Hier steht eine Anzeige.}

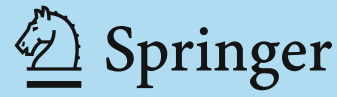

